\title{
The Ambiguous "Internal Carotid Artery" - Ultrasound Diagnosis of One Case of Congenital Absence of the Internal Carotid Artery
}

\section{Gang Zhong}

Hunan Provincial People's Hospital(The first-affiliated hospital of Hunan normal university) Jing Ning

Hunan Provincial People's Hospital(The first-affiliated hospital of Hunan normal university)

\section{Xiangdang Long ( $\square$ hnlxd1997@126.com )}

Hunan Provincial People's Hospital(The first-affiliated hospital of Hunan normal university)

\section{Juanjuan Xie}

Hunan Provincial People's Hospital(The first-affiliated hospital of Hunan normal university)

\section{Kun Ao}

Hunan Provincial People's Hospital(The first-affiliated hospital of Hunan normal university)

\section{Fang Liu}

Hunan Provincial People's Hospital(The first-affiliated hospital of Hunan normal university)

\section{Mengyi Huang}

Hunan Provincial People's Hospital(The first-affiliated hospital of Hunan normal university)

\section{Yu Zhuo}

Hunan Provincial People's Hospital(The first-affiliated hospital of Hunan normal university)

\section{Qiaorong Li}

Hunan Provincial People's Hospital(The first-affiliated hospital of Hunan normal university)

\section{Qiongli Wen}

Hunan Provincial People's Hospital(The first-affiliated hospital of Hunan normal university)

\section{Qiuyi Di}

Hunan Provincial People's Hospital(The first-affiliated hospital of Hunan normal university)

\section{Case Report}

Keywords: congenital absence of the internal carotid artery, aneurysm, diagnosis, ultrasound

Posted Date: June 24th, 2022

DOI: https://doi.org/10.21203/rs.3.rs-1031069/v2 
License: (c) (i) This work is licensed under a Creative Commons Attribution 4.0 International License. Read Full License 


\section{Abstract}

Background: Congenital absence of the internal carotid artery (ICA) is a very rare congenital anomaly of the ICA. Most reports in the literature are of individual cases, and most were diagnosed by computed tomographic angiography (CTA) or digital subtraction angiography (DSA). There are few reports on the diagnosis of congenital absence of the ICA by carotid Doppler ultrasound (CDUS).

Case presentation: A 61-year-old male patient who was admitted to the hospital because of dizziness and showed no abnormality on CDUS conducted at another hospital. CDUS conducted at our hospital found maldevelopment of the right common carotid artery (RCCA) with normal a blood flow velocity and spectrum. A high-resistance frequency spectrum could be detected in the "ICA" that continued directly from the RCCA, and two adjacent small blood vessel branches were also observed. Superficial temporal artery tap and tracing of the courses of the small blood vessels confirmed that the RCCA directly continued to the external carotid artery (ECA). The final diagnosis was congenital absence of the ICA, which was confirmed during the clipping of an anterior communicating artery (ACoA) aneurysm.

Conclusion: CDUS, as a noninvasive and rapid screening tool for cervical vascular diseases, offers a new approach for the diagnosis of congenital absence of the ICA.

\section{Background}

The internal carotid artery (ICA) is one of the main arteries that supplies blood to the brain and one of the most stable blood vessels in human body. Congenital anatomical variation rarely occurs, and congenital absence of the ICA is even rarer, with an incidence rate of less than $0.01 \%^{[1-3]}$. Congenital absence of the ICA often has no obvious clinical symptoms; therefore, it is often discovered by chance during physical examination. However, ICA absence is often accompanied by the formation of an intracranial aneurysm [1], which may lead to serious complications, such as aneurysm rupture and bleeding and even death. Therefore, early and accurate diagnosis of congenital absence of the ICA is an important measure to prevent stroke. Carotid Doppler ultrasound (CDUS), the most commonly used screening tool for carotid artery diseases, allows the dynamic observation of the courses, lumen diameters and wall structures of the carotid arteries; provides information about the blood flow velocity and spectrum; and aids in the evaluation of hemodynamic changes and the diagnosis of congenital dysplasia. Congenital absence of the ICA is rare and is usually diagnosed by computed tomographic angiography (CTA) or digital subtraction angiography (DSA) in the clinic. Radiologists may not have adequate knowledge about this disease and its ultrasonic characteristics. Therefore, missed diagnosis may occur.

\section{Case Presentation}

The patient was a 61-year-old male who was admitted to hospital due to dizziness for more than half a month. He was known to have had hypertension for more than half a month and denied a history of heart disease, diabetes, cerebrovascular disease or mental illness. He had a 40-year history of drinking history 
and smoking history. Physical examination showed that the bilateral frontal lines were symmetrical, and the nasolabial grooves showed no shallowing. No skewness in the corner of the mouth was observed. No eyelid ptosis was found. Bilateral facial sensation, shallow sensation and muscle tension of trunk and limbs revealed no abnormalities. Bilateral pathological signs were negative. CDUS performed at an outside hospital showed no abnormality.

CDUS conducted at our hospital showed uneven intima-media thickening of the bilateral common carotid arteries (CCAs) and a "bifurcation structure" comprising a directly continuing "ICA" and two adjacent small blood vessel branches observed in the right common carotid artery (RCCA) (Fig.1a). The measured values of the RCCA suggested maldevelopment with normal blood flow velocity and spectrum (Fig.1b). The right "ICA" showed a high-resistance spectrum, indicated by sawtooth-like changes during diastole upon temporal artery tap(Fig.1C). After tracing the course of two small blood vessels, we was confirmed that the right "ICA" was actually the external carotid artery (ECA), the RCCA continued directly to the ECA, and the two small blood vessels were branch arteries of the ECA. Therefore, congenital absence of the ICA was considered.

CTA (Fig.2) showed that the right ICA (RICA) was absent, the RCCA had no obvious bifurcation, the left CCA (LCCA) and the left ICA (LICA) were thick, LICA C7 was slightly calcified, right anterior cerebral artery (RACA) A1 was absent, the RACA originated from the left ACA (LACA), and the right middle cerebral artery (RMCA) originated from an LICA branch. A nodular aneurysm with a diameter of approximately $3.7 \mathrm{~mm}$ could be observed in the anterior communicating artery (ACoA). DSA (Fig.3) revealed an ACoA aneurysm. The RICA was not developed, the RACA was supplied by the LACA through the ACoA, and the RMCA was supplied by the LICA through an anastomotic branch between the cavernous sinuses.

After relevant preoperative examinations were performed, the patient underwent right craniotomy and clipping of the aneurysm under general anesthesia 8 days after admission. During the operation, an aneurysm approximately $4 \times 3 \mathrm{~mm}$ in size was observed in the ACoA complex of the brain, and the RICA was not explored. It was observed that the LICA sent out a lateral branch vessel that connected with the RMCA above the tuberculum sellae and below the dura mater(Fig.4). Postoperative diagnosis indicated 1. ACoA aneurysm; 2. Congenital absence of the RICA.

\section{Discussion}

At present, the pathogenesis of congenital absence of the ICA is unclear. The disease may be related to the arrested development or secondary degeneration of the ICA during embryonic development. Published studies ${ }^{[1-3]}$ have reported that the incidence of congenital absence of the ICA is less than $0.01 \%$; unilateral absence of ICA is more common, with a ratio of left absence to right absence of approximately 3:1. Bilateral absence accounts for less than $10 \%$ of all cases of congenital absence of the ICA. Patients with congenital absence of the ICA often have no clinical symptoms due to compensation by the collateral circulation of Willis circle. Some patients may experience noncharacteristic symptoms such as tinnitus, dizziness, headache, etc., and a small number of patients may be admitted to the hospital due to 
aneurysm rupture or epilepsy ${ }^{[4]}$. Lie ${ }^{[5]}$ divided congenital absence of ICA into 6 types according to the compensatory pathways of collateral circulation. In Type A (unilateral absence of the ICA), the most common type encountered in clinical practice, the affected ACA is supplied by the contralateral ACA via the ACoA, and the affected MCA is supplied by the ipsilateral posterior cerebral artery (PCA). In Type B (unilateral absence of ICA), the affected ACA and MCA are supplied by the contralateral ACA via the ACoA. In Type C (bilateral absence of ICA), the bilateral ACAs and MCAs pass through the posterior communicating artery (PCOA) to supply blood from the posterior cerebral circulation. In Type D (unilateral absence of ICA), the siphon section of the affected ICA is supplied by the contralateral ICA via the formation of an anastomotic branch between the cavernous sinuses. In Type E, the bilateral small ACAs are supplied by the bilateral maldeveloped ICAs, and the bilateral MCAs are supplied by the PCA via the PCoA. In Type F, the ACA and MCA on the affected side are supplied by anastomosis of the ECA-internal maxillary artery to the skull base, that is, the skull base microvascular network. In the present case, the RACA A1 was absent, the RACA was supplied by the LACA via the ACoA, and the RMCA was supplied by the LICA through an anastomotic branch between the cavernous sinuses; this corresponds to Type $D$ in the Lie classification and is very rare. According to the study results of Jesse et al. ${ }^{[6]}$, the incidence of intracranial aneurysms in the general population is approximately $2 \%-4 \%$, while the incidence of intracranial aneurysms in patients with congenital absence of the ICA is as high as $25 \%-43 \%$, which may be due to congenital dysplasia of the vascular wall or abnormal hemodynamics ${ }^{[7]}$. Therefore, early and accurate diagnosis of congenital absence of the ICA and regular monitoring of the occurrence and development of intracranial aneurysms are important for preventing cerebral hemorrhage and subarachnoid hemorrhage ${ }^{[8]}$.

CDUS is the most commonly used screening tool for cervical vascular diseases. It offers the advantages of high resolution, high safety, convenience and low cost and plays an important role in the diagnosis of congenital dysplasia of the carotid artery. Because congenital absence of the ICA is rare, most reports are individual cases, and most cases are diagnosed by CTA or DSA, there are few reports on the diagnosis of congenital absence of the ICA by CDUS. Radiologists may not have adequate knowledge about this disease and its ultrasonic characteristics, which may lead to missed diagnosis. In the present case, CDUS conducted at an outside hospital showed no abnormality. During examination, we found that the patient's RCCA was maldeveloped, but its blood flow velocity and spectrum were normal. When we saw the bifurcated structure of the RCCA, we mistakenly thought that the thicker blood vessel that continued directly was the ICA. Pulse Doppler showed that its resistance index $(\mathrm{RI})$ was significantly higher than that of the LICA and slightly higher than that of the RCCA; therefore, it was suspected to be the ECA. A superficial temporal artery tap was carried out, and the result showed sawtooth-like changes in the diastolic spectrum. Careful scanning revealed that there were two adjacent small blood vessel branches at the "bifurcation". By tracing their courses, we observed that the first branch was the superior thyroid artery, and the other branch went towards the side of the head. Therefore, it was confirmed that the RCCA continued directly to the ECA. The patient was finally diagnosed with congenital absence of the ICA. 
When absence of the ICA is suspected on ultrasonography, it should be differentiated from ICA occlusion. Occlusion of the ICA is most often caused by the formation of an atherosclerotic plaque, but it can also be observed in cases of arteritis, cardiogenic embolism, etc. Ultrasound can not only show the wall structure of the occluded ICA but can sometimes be used to explore the stump of the ICA ${ }^{[9]}$. Transthoracic echocardiogram (TTE) can be performed when cardiogenic embolism is considered. Kaya et al. ${ }^{[10]}$ suggested that dysplasia of the CCA on the affected side is an important clue in the ultrasonic diagnosis of congenital absence of the ICA. Yilmaz et al. ${ }^{[11]}$ found that most patients with ICA occlusion showed decreased peak systolic velocity and significantly decreased peak diastolic velocity, and some even presented with a reversed spectrum in the affected CCA. However, the peak systolic and diastolic velocity of the CCA in congenital absence of the ICA is normal. Therefore, ICA occlusion can basically be ruled out when the ICA is not visible and the CCA on the affected side shows a low-resistance spectrum. In this case, the RCCA was maldeveloped, and the spectrum, blood flow velocity and resistance of the bilateral CCAs were very similar, without the obvious wall structure of the RICA. Therefore, it was considered that the RICA was absent rather than occluded. In addition, because the ICA forms earlier than the skull base, CT of the skull base can also assist in the diagnosis of congenital absence of the ICA if the bony carotid canal is maldeveloped or not developed ${ }^{[7]}$.

\section{Conclusion}

CDUS, as a noninvasive and rapid screening tool for cervical vascular diseases, offers a new approach for the diagnosis of congenital absence of the ICA. Radiologists should improve their understanding of the disease and its corresponding ultrasonic characteristics. When maldevelopment of the CCA is found on one side and pulse Doppler shows that the blood flow velocity and spectrum are normal, and the ipsilateral ICA is not visible, the possibility of congenital absence of the ICA should be considered.

\section{Abbreviations}

ICA: Internal carotid artery; CTA: Computed tomographic angiography; DSA: Digital subtraction angiography; CDUS: Carotid Doppler ultrasound; RCCA: Right common carotid artery; LCCA: Left common carotid artery; ECA: External carotid artery; ACoA: Anterior communicating artery; CCA: Common carotid arterie; RICA: Right internal carotid artery; LICA: Left internal carotid artery; ACA: Anterior cerebral artery; RACA: Right anterior cerebral artery; LACA: Left anterior cerebral artery; MCA: Middle cerebral artery; RMCA: Right middle cerebral artery; PCA: Posterior cerebral artery; PCoA: Posterior communicating artery; RI: Resistance index; TTE: Transthoracic echocardiogram.

\section{Declarations}

Ethics approval and consent to participate: Not applicable.

Consent for publication: Written informed consent was obtained from the patient. 
Availability of data and materials: The datasets used and analysed during the current study are available from the corresponding author on reasonable request.

Competing interests: The authors declare no conflicts of interest regarding the publication of this study.

Funding: Not applicable.

Author's contributions: All authors provided intellectual contribution to this paper. ZG and NJ designed and wrote this paper. LXD involved in case review and revision of the manuscript. XJJ, AK, LF, ZY, LQR, WQL and DQY collected the clinical data, including CDUS and CT images. HMY was the main surgeon for the operation and conceived of the study.

Acknowledgments $\square$ Not applicable.

\section{References}

1. Taşar M, Yetişer S, Taşar A, Uğurel S, Gönül E, Sağlam M. Congenital absence or hypoplasia of the carotid artery: radioclinical issues. Am J Otolaryngol. 2004 Sep-Oct;25(5):339-49.

2. Li S, Hooda K, Gupta N, Kumar Y. Internal carotid artery agenesis: A case report and review of the literature. Neuroradiol J. 2017 Apr;30(2):186-191.

3. Akdemir Aktaş H, Farımaz M, Fırat A, Göçmen R. Rare congenital anomalies of the internal carotid artery: anatomic and radiologic aspects of three cases and review of the literature. Surg Radiol Anat. 2020 Nov;42(11):1363-1370.

4. Lie TA. Congenital anomalies of the carotid arteries. Amsterdam: Excerpta Medica. 1968:35-51.

5. Chen J, Raden M, Lin C. Congenital absence of the internal carotid artery with intercavernous anastomosis. Radio/ Case Rep. 2019 Jun 10;14(8):1021-1026.

6. Hebant B, Gerardin E, Delpierre C, Mereaux JL, Quesney G, Lefaucheur R. A case of right internal carotid agenesis with associated transcavernous anastomosis revealed by transient ischemic attack. Surg Radiol Anat. 2020 Feb;42(2):207-210.

7. Guerreiro C, Basílio G, Sequeira P. Congenital Absence of the Internal Carotid Artery With Intercavernous Anastomosis. World Neurosurg. 2020 Nov;143:68-69.

8. Huang Wei, Chen Wen, Li Jun. A Case Report of Congenital Absence of Internal Carotid Artery Complicated with Intracranial Arteriovenous Malformation and Literature Review. Chinese Journal of Neurosurgery. 2019, 35 (1): 90-91.

9. Kaya O, Yilmaz C, Gulek B, Soker G, Cikman G, Inan I, Demirduzen S. An important clue in the sonographic diagnosis of internal carotid artery agenesis: ipsilateral common carotid artery hypoplasia. Case Rep Radiol. 2014; 2014:516456.

10. Yilmaz C, Utebay B, Kalaycioglu S, Onat G, Solak A. Non-visualization of the internal carotid artery with a normal ipsilateral common carotid artery Doppler waveform: a finding suggesting congenital absence of the ICA on colour Doppler ultrasound. Br J Radiol. 2006 Sep;79(945):e108-11. 


\section{Table}

Table.1

Ultrasound measurements of the bilateral carotid artery.

\begin{tabular}{|c|c|c|c|c|c|c|c|c|}
\hline & Right & & & & Left & & & \\
\hline & $\begin{array}{l}\text { Diameter } \\
\bigotimes \mathrm{mm} \rrbracket\end{array}$ & 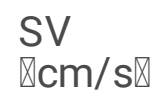 & 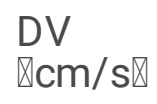 & $\mathrm{RI}$ & $\begin{array}{l}\text { Diameter } \\
\bigotimes \mathrm{mm} \rrbracket\end{array}$ & $\begin{array}{l}\mathrm{PV} \\
\bigotimes \mathrm{cm} / \mathrm{s} \rrbracket\end{array}$ & $\begin{array}{l}\mathrm{DV} \\
\text { \cm/s》 }\end{array}$ & RI \\
\hline CCA & 5.3 & 80 & 21 & 0.74 & 9.1 & 79 & 18 & 0.77 \\
\hline ICA & I & & & & 7.7 & 47 & 14 & 0.70 \\
\hline ECA & 4.6 & 80 & 19 & 0.76 & 5.1 & 65 & 7 & 0.89 \\
\hline
\end{tabular}

CCA indicates right common carotid artery; ICA, internal carotid artery; ECA, external carotid artery; SV, systolic velocity; DV, diastolic velocity; RI, resistance index.

\section{Figures}

\section{Figure 1}

\section{Carotid Doppler ultrasound.}

A. A "bifurcated structure" of the RCCA - a directly continuing "ICA" and two adjacent small blood vessel branches (arrows). B. Pulsed Doppler ultrasound shows that the blood flow velocity and spectrum of the RCCA are normal during systole and diastole. C. Pulsed Doppler ultrasound shows an obvious anterograde diastolic blood flow spectrum of the right "ICA" and typical diastolic sawtooth-like changes during superficial temporal artery tap. RCCA indicates right common carotid artery; ICA, internal carotid artery. 


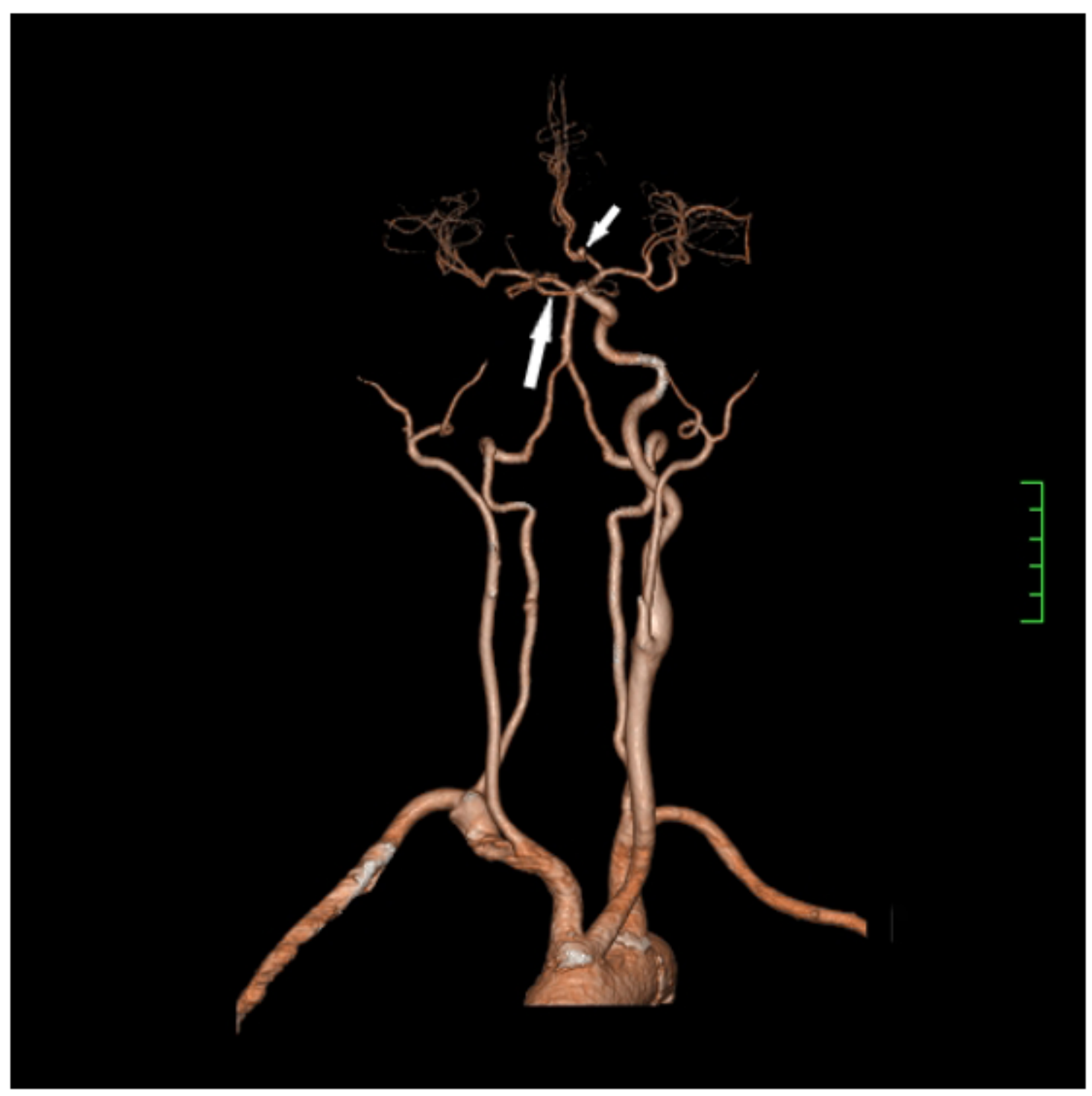

Figure 2

\section{Threedimensional computerized tomography angiography.}

The RCCA is thinner than the LCCA and continues directly to the ECA. The LICA connects with the RMCA via an anastomotic branch between the cavernous sinuses (long arrow), and the LACA connects with the RACA via the ACoA, showing an ACoA aneurysm (short arrow). RCCA indicates right common carotid artery; LCCA, left common carotid artery; ECA, external carotid artery; LICA, left internal carotid artery; RMCA, right middle cerebral artery; LACA, left anterior cerebral artery; RACA, right anterior cerebral artery; ACoA, anterior communicating artery.

Figure 3 


\section{Digital subtraction angiography.}

The RCCA is obviously thinner than the LCCA, and no bifurcated structure is found. B. The RCCA continues directly to the ECA. C. The RMCA is supplied by the LICA via the anastomotic branch between the cavernous sinuses (long arrow), and the RACA is supplied by the LACA via ACoA, showing an ACoA aneurysm (short arrow). RCCA indicates right common carotid artery; LCCA, left common carotid artery; ECA, external carotid artery; RMCA, right middle cerebral artery; LICA, left internal carotid artery; RACA, right anterior cerebral artery; LACA, left anterior cerebral artery; ACoA, anterior communicating artery.

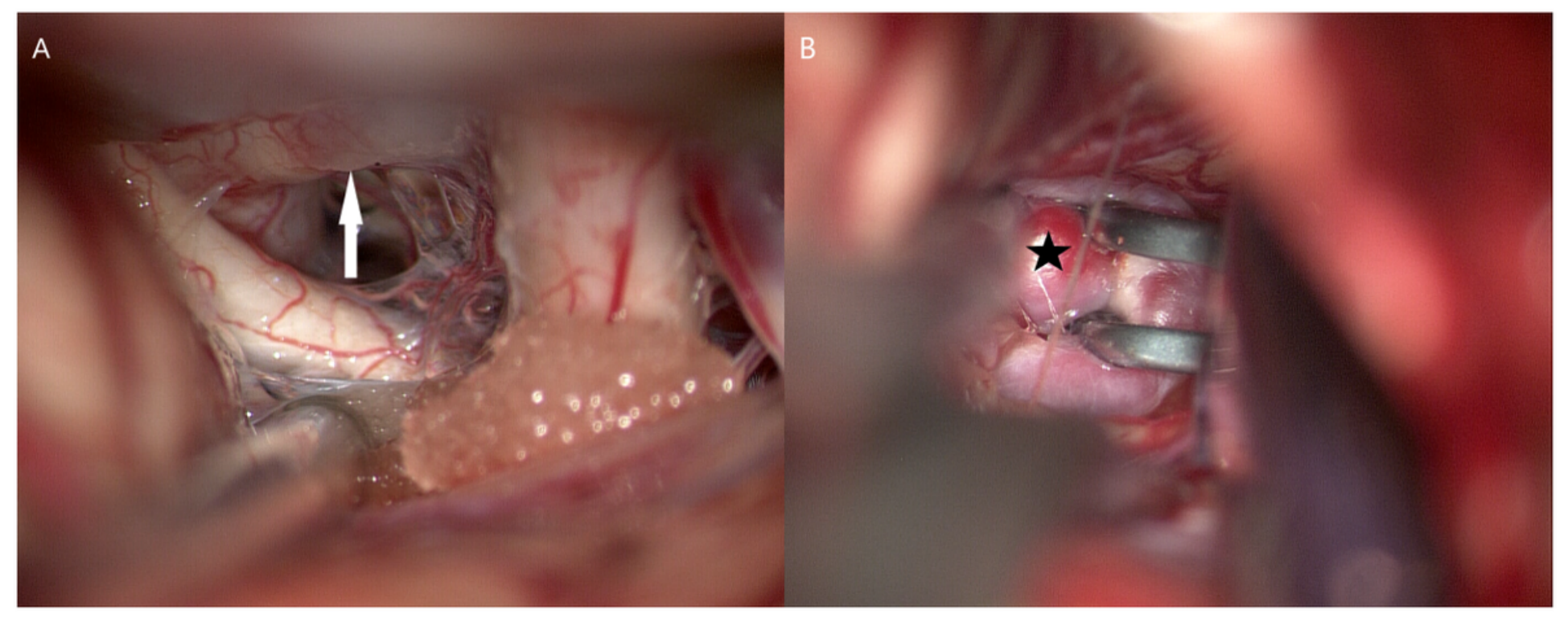

\section{Figure 4}

\section{Intraoperative macroscopic view.}

A. The anastomotic branch between the cavernous sinuses (arrow) originates from the LICA and travels under the dura mater and above the tuberculum sellae. B. The ACoA aneurysm(asterisk). LICA indicates left internal carotid artery; ACoA, anterior communicating artery. 